\title{
Molecular pattern of IncRNAs in hepatocellular carcinoma
}

\author{
Haoming Mai ${ }^{1,2}$, Bin Zhou ${ }^{1,2}$, Li Liu ${ }^{1,2}$, Fu Yang ${ }^{3}$, Carly Conran ${ }^{4}$, Yuan Ji ${ }^{5}$, Jinlin Hou ${ }^{1,2}$ and Deke Jiang ${ }^{1,2^{*}}$
}

\begin{abstract}
Hepatocellular carcinoma (HCC) is one of the most notable lethal malignancies worldwide. However, the molecular mechanisms involved in the initiation and progression of this disease remain poorly understood. Over the past decade, many studies have demonstrated the important regulatory roles of long non-coding RNAs (IncRNAs) in HCC. Here, we comprehensively review recent discoveries regarding HCC-associated IncRNA functions, which we have classified and described according to their mechanism models.
\end{abstract}

Keywords: IncRNA, Molecular pattern, Regulation of biological processes, Hepatocellular carcinoma, Wellcharacterized mechanism

\section{Background}

According to the World Health Organization (WHO), liver cancer is the sixth most common malignancy worldwide. In 2018, there were approximately 841,000 new liver cancer patients in the world, and approximately $83 \%$ occurred in Eastern Asia. The prognosis of liver cancer is very poor, and the mortality rate is high, resulting in approximately 781,000 deaths in 2018, which is the fourth most common cause of cancer deaths (approximately $8.2 \%$ of cancer deaths). Hepatocellular carcinoma (HCC) is the primary form of liver cancer, comprising $75-85 \%$ of cases [1]. Prognosis is closely related to early diagnosis of the disease. However, HCC has a lengthy subclinical stage, with an insidious initiation and progression that is often difficult to detect. Thus, most HCC patients are diagnosed at an advanced stage, and treatment options at this stage are limited. Moreover, the development of HCC is a multi-step process involving many gene networks and changes in signaling pathways, and many of these pathways remain to be elucidated [2]. It is therefore important to decipher the molecular pathological mechanism of HCC to better

\footnotetext{
* Correspondence: dekejiang17@smu.edu.cn

${ }^{1}$ State Key Laboratory of Organ Failure Research, Guangdong Key Laboratory of Viral Hepatitis Research, Institute of Liver Diseases Research of Guangdong Province, Guangzhou, China

${ }^{2}$ Department of Infectious Diseases and Hepatology Unit, Nanfang Hospital, Southern Medical University, Guangzhou 510515, China

Full list of author information is available at the end of the article
}

assess patient prognosis and identify, or develop, optimal therapies.

According to the biological central dogma, RNAs are considered to be a template for protein translation (mRNAs) and the infrastructure supporting this process (tRNAs and rRNAs) [3]. However, knowledge gained from human genome sequencing has challenged this rule [4]. The number of "genes" estimated by express sequence tag data in the year 2000 ranged from 45,000 to 140,000 [5]. Later, the International Human Genome Sequencing Consortium proposed that the human genome has only 20,000 to 25,000 protein-coding genes [6]. Soon after, the ENCyclopedia Of DNA Elements (ENCODE) project noted that only $2 \%$ of the human genome is encoded into protein, though $74.7 \%$ of the human genome is transcribed, with no cell line expressing more than $56.7 \%$ of this human transcriptome collection [7]. Furthermore, $62 \%$ of the human genome encodes long RNA molecules ( $>200$ nucleotides) [8]. These pervasive studies brought to light the functional importance of this so-called "junk" DNA. Non-coding RNAs (ncRNAs) are not just "transcriptional noise," as previously thought, which fundamentally changes our interpretation of the genome and transcriptome $[9,10]$.

The development of high-throughput sequencing technology, such as next generation sequencing, has led to the discovery of a large number of ncRNAs, of which long ncRNAs are the largest, attracting great attention in the past decade. Integrated analysis of RNA-seq data

(C) The Author(s). 2019 Open Access This article is distributed under the terms of the Creative Commons Attribution 4.0 International License (http://creativecommons.org/licenses/by/4.0/), which permits unrestricted use, distribution, and reproduction in any medium, provided you give appropriate credit to the original author(s) and the source, provide a link to the Creative Commons license, and indicate if changes were made. The Creative Commons Public Domain Dedication waiver (http://creativecommons.org/publicdomain/zero/1.0/) applies to the data made available in this article, unless otherwise stated. 
revealed that $68 \%$ of human transcripts are lncRNAs with an estimated number of 55,000 to 60,000 [11]. LncRNAs are generally defined as transcripts longer than 200 nucleotides that do not have protein-coding potential; this relatively arbitrary cutoff distinguishes small ncRNAs from lncRNAs. Furthermore, lncRNAs are more similar to mRNAs than to other ncRNAs. First, the genome loci of IncRNA has a similar chromatin state to the mRNA genome loci, from which lncRNAs are transcribed by RNA polymerase II (Pol II) [12]. In addition, like mRNAs, lncRNAs are often polyadenylated, $5^{\prime}$-capped and spliced [13]. Therefore, analysis of whether open reading frames have protein-coding potential is an important means by which to distinguish lncRNAs from mRNAs. Relatively speaking, lncRNAs have lower expression levels, shorter transcripts, poorer sequence conservation and more nuclear enrichment than mRNAs [14].

There is no standard system for the identification and classification of lncRNA and their functions. This is likely due to the sheer number of lncRNAs, as well as their complex structures. In the past few years, reviews have attempted to classify lncRNAs by various molecular functions, including: (1) lncRNAs are divided into cis-acting lncRNAs and trans-acting lncRNAs by their genomic locus [15]. (2) lncRNAs are distinguished by the biological processes in which IncRNAs are involved [16, 17]. (3) Cancer-associated IncRNA are classified by their effects on cancer phenotype [18]. (4) HCC-associated lncRNAs are separated by their related signaling pathways [19]. In addition, lncRNAs can function via direct interaction with DNA, RNA and protein, making them versatile within biological processes. However, although lncRNAs have been extensively studied in recent years, the role of lncRNAs in the initiation and development of HCC remains to be characterized. Just as with lncRNAs in other fields, the identification and classification of these mysterious molecules in HCC are extremely challenging, ambiguous and full of exceptions. This review aims to classify the molecular mechanisms of HCC-associated lncRNAs comprehensively and clearly by combining lncRNA modes of molecular interaction with their involved biological processes (Additional file 1: Table S1).

\section{Classification of modes of molecular interaction of HCC-associated IncRNAs}

Despite the diverse functions of lncRNAs [15], previous studies have shown that the primary way in which HCC-associated lncRNAs exert their biological roles is via interactions with DNA, RNA and proteins. To facilitate the understanding and categorization of these molecular modes of interaction, rare molecular interaction modes that have been elucidated under other physiological and pathological conditions would be excluded in this paper. The three molecular interactions described here represent the IncRNA molecular mechanisms that have been validated in HCC cell lines or tissues. (1) Sequester: The interactions of lncRNAs with DNA, RNA or proteins cause these molecules to be isolated from the original interacting molecules, thereby preventing the original interaction. (2) Scaffold: LncRNAs interact with various molecules to create linkages for these molecules and facilitate interaction between these molecules. (3) Guide (can be considered as a special case of scaffold): LncRNAs bind to transcription factors or chromatin-modifying complexes, directing these molecules to specific genomic sites to promote or inhibit the transcription of related genes (Additional file 1: Table S1).

\section{HCC-associated IncRNAs participate in various biological processes}

The physiological and pathological effects of lncRNAs are achieved primarily through gene expression regulation. Transcription and translation are key phases of these biological processes $[15,16]$. In HCC, lncRNAs are involved specifically in epigenetic regulation, transcription factor regulation, post-transcriptional regulation, and protein degradation. In addition, some lncRNAs affect protein modification and protein complex modulation in HCC; however, this classification is often rather subjective, as HCC-associated IncRNAs always regulate protein degradation by affecting protein ubiquitination, which is a type of protein modification [20-25]. Conversely, some protein modifications of transcription factors are regulated by HCC-associated lncRNAs [26, 27]. Yan et al. suggested that protein complex assembly affected by lncRNAs can influence protein function, thereby regulating the corresponding signaling pathway [28]. Thus, the molecular interaction modes of HCC-associated IncRNAs and the regulation of biological processes are diverse, and many details remain unclear. In this review, we propose a comprehensive, yet simple, method of combining modes of molecular interactions and biological processes to analyze the mechanisms by which lncRNAs exert their effects on HCC risk (Additional file 1: Table S1).

\section{HCC-associated IncRNAs in epigenetic regulation}

A suitable chromatin state is crucial for gene expression. The relationship between chromatin state and nucleosome histone modification has been studied extensively. As an epigenetic repressor, polycomb repressive complex 2 (PRC2) can inhibit the transcription of various genes, and accelerate the development of HCC, through histone $\mathrm{H} 3$ lysine 27 (H3K27) trimethylation. However, as a trans-acting regulator, the mechanism by which PRC2 interacts with its target genes remains to be characterized [29]. 
In recent years, studies have indicated that $20 \%$ of lncRNAs can bind to PRC2 [30]. PRC2-associated lncRNAs have also been confirmed in HCC, among which the most recognized lncRNA may be HOX transcript antisense RNA (HOTAIR) [30, 31]. By directly interacting with the core subunit component enhancer of zeste homolog 2 (EZH2) of PRC2, HOTAIR can recruit PRC2 to the promoter region of miR-218, resulting in reduced expression of miR-218 (Fig. 1a). MiR-218 and Bmi-1 mRNA have a perfect seed pairing, which inhibits the activity of the P14ARF and P16Ink4a signaling pathway by reducing the translation of Bmi-1 [32]. According to the competing endogenous RNA (ceRNA) hypothesis, the microRNA binding sites on IncRNA or mRNA can titrate microRNAs (miRNAs) and regulate microRNA availability. Since it is easy to predict computationally, the IncRNA-miRNA-mRNA regulation axis is more likely to be interpreted by the ceRNA hypothesis [33] Therefore, the mechanism by which lncRNAs inhibit miRNAs epigenetically is relatively inspiring. The molecular mechanism proposed by this study is novel and instructive on how to unravel the correlation between lncRNA and miRNA expression. Additionally, various HCC-associated lncRNAs have been shown to directly interact with EZH2 and inhibit the expression of different genes through a similar histone modification mechanism [34-39]. However, several studies have suggested the interaction between PRC2 and lncRNAs may also be promiscuous $[40,41]$. Besides, HOTAIR has been reported to widely regulate the genome occupancy of PRC2, thus making it inappropriate to interpret this phenomenon simply in terms of DNA-RNA sequence
A

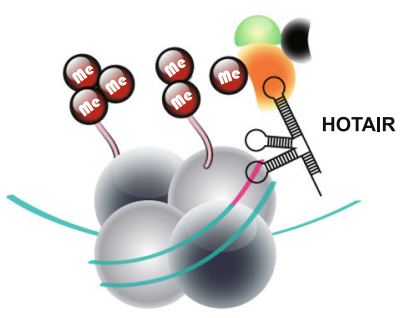

B

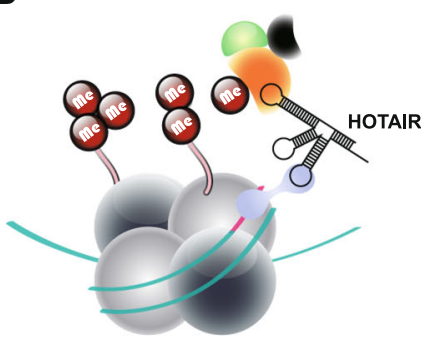

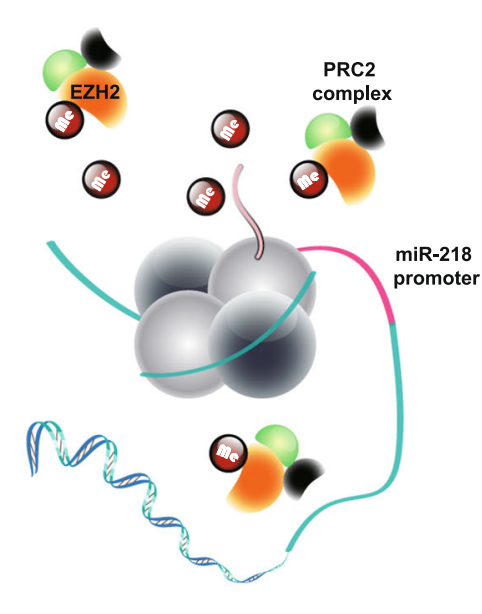

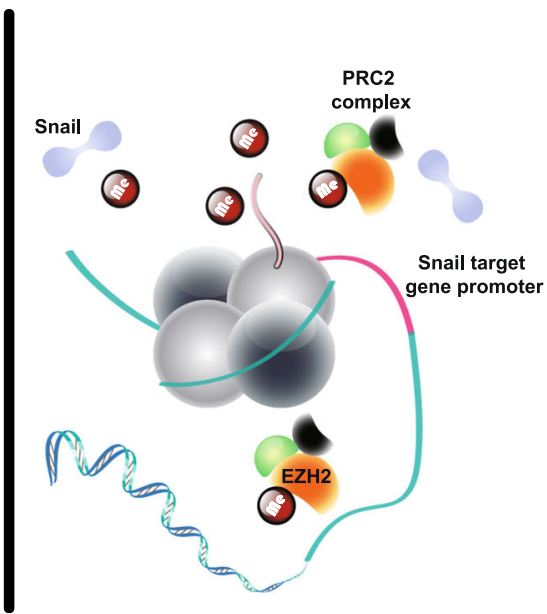

Fig. 1 HCC-associated IncRNAs in histone modification. a HCC-associated IncRNAs recruit histone modifiers to regulate gene expression. Left panel: HOTAIR suppresses miR-218 expression by recruiting PRC2 to miR-218 promoter, which induces chromatin remodeling and H3K27 trimethylation. Right panel: decreased HORAIR inhibits PRC2-mediated miR-218 transcription suppression. b HCC-associated IncRNAs act as scaffold to bridge histone modifiers to regulate gene expression. Left panel: HOTAIR bridges PRC2 complex and Snail, which leads to H3K27 trimethylation in promoter region of Snail target genes. Right panel: decreased HORAIR inhibits PRC2-mediated transcription suppression of Snail target genes 
complementarity [31], so this recruitment of $\mathrm{PRC} 2$ is likely to involve other molecules and mechanisms.

In fact, a previous study has proposed that the Snail protein can directly recruit PRC2 to epithelial targets [42]. During epithelial to mesenchymal (EMT) in HCC, HOTAIR acts as a scaffold to bridge PRC2 and Snail, which suppresses expression of HNF4a (hepatocyte nuclear factor 4, alpha), HNF1a (HNF1 homeobox A) and E-cadherin by Snail-dependent manner (Fig. 1b). The promoter regions of these genes contain E-box, a consensus Snail-binding sequence. When Snail is knocked down, it can significantly impair the HOTAIR-repressive activity on transcription of these Snail target genes [42].

HOTAIR has long been considered a risk factor for HCC $[43,44]$. Interestingly, though, Zhang et al. found that the expressions of a human cancer stem cell marker, epithelial cell adhesion molecule (EpCAM), and pluripotent genes were increased by knocking down HOTAIR through siRNA transfection, thus making HOTAIR do not function as an oncogene [20]. Further, this research has shown that RNA Helicase DEAD Box Protein 5 (DDX5) is involved in this biologic process. Decreased levels of DDX5 suggests a poor prognosis for HCC patients. DDX5 can bind to HOTAIR and subunit suppressor of zeste 12 homolog (SUZ12), a PRC2 subunit, displacing an E3 ligase, Mex-3 RNA-binding family member B (Mex3b), from HOTAIR to inhibit Mex-3b-mediated SUZ12 degradation (Fig. 5b). DDX5 is thereby involved in the transcription inhibition of EpCAM and other pluripotency genes via the HOTAIR-PRC2 complex. Hepatitis B virus (HBV) infection downregulates DDX5 expression by the $\mathrm{HBx}$ protein, resulting in increased expression of the above-mentioned pluripotent genes. Therefore, HOTAIR regulates transcription inhibition and protein degradation simultaneously. Finally, two risk factors of HCC, HOTAIR and HBV infection, jointly promote the development of HCC [20]. Hence, some histone modifications in chromatin-modifying complexes in HCC are not only dependent on lncRNA, and the true mechanisms remain to be elucidated.

In addition to HOTAIR, some HCC-associated, lncRNA-mediated epigenetic regulations also demonstrate their complexity. For instance, IncRNA Gradually Increased During Hepatocarcinogenesis (GIHCG) not only regulates the transcription of $\mathrm{miR}-200 \mathrm{a} / \mathrm{b} / 429$ via PRC2-mediated histone H3 lysine 27 trimethylation, but also methylates the histone promoter regions of these genes via DNA methyltransferase 1 (DNMT1). Thus, both regulatory mechanisms synergistically inhibit the expression of these genes [45]. Moreover, in addition to sharing a bidirectional promoter with retinoblastoma gene 1 (RB1), linc00441 also induces methylation of RB1 in the promoter region by recruiting DNA methyltransferase 3 (DNMT3), which reduces RB1 transcription (Fig. 2) [46].
In addition to regulating gene transcription by histone methylation and DNA methylation, HCC-associated lncRNAs, including $\mathrm{H} 19$ and GPC3 antisense RNA 1 (GPC3-AS1), also alter histone acetylation to influence HCC progression [47, 48]. Notably, lncTCF7 promotes liver CSC self-renewal by guiding the SWItch/Sucrose Non-Fermentable (SWI/SNF) complex to initiate transcription factor 7 .

(TCF7) expression [49]. The SWI/SNF complex uses energy produced by ATP hydrolysis to directly mobilize nucleosomes and remodel chromatin, making it a distinctive epigenetic regulator [50]. Coupled with the above-described histone methylation, acetylation and DNA methylation, HCC-associated lncRNAs exhibit diverse capabilities in epigenetic regulation (Figs. 1 and 2). And the molecular interaction modes mentioned in this section are mainly guide and scaffold, further research in this field may help to characterize more mechanisms of lncRNA-based epigenetic regulation in HCC.

\section{HCC-associated IncRNAs in transcription factor regulation}

In the nucleus, in addition to epigenetic regulation, lncRNAs can directly affect transcription factor function. Among them, lncSox4 (also known as cancer susceptibility 15, CASC15) is a nucleus-enriched lncRNA that is highly expressed in liver cancer and liver tumor-initiating cells (TIC). Mechanism investigation found that lncSox4 binds the sex determining region Y-box 4 (Sox4) promoter and recruits signal transducer and activator of transcription 3 (STAT3) to promote Sox4 expression, which is required for liver TIC self-renewal (Fig. 3a) [51]. Another HCC-associated lncRNA that regulates transcription factor function is lncWDR26; however, the result of this interaction is the transcriptional repression of WD repeat domain 26 (WDR26). As a tumor suppressor, SIX homeobox 3 (SIX3) can inhibit the expression of some metastasis and proliferation-related genes [52]. As a down-regulated lncRNA in HCC, the lncWDR26 recruits SIX3 to WDR26 promoter regions and represses WDR26 transcription [53]. In general, HCC-associated lncRNAs can directly guide transcription factors to their specific binding sites, leading to transcriptional activation or inhibition of related genes (Fig. 3a).

LncRNAs can also prevent transcription factors binding to their target promoters by interaction with them. For example, by comparing the transcriptome of HepG2 cells and HBV transgenic HepG2-4D14 cells, it was found that lnc-HUR1 transcription was enhanced by HBV-encoded HBx. Mechanistically, p53 is detained by lnc-HUR1, resulting in reduced promoter occupancy of the target gene, such as p21 and Bax (BAX). In conclusion, lnc-HUR1 can inhibit p53-promoted transcription 


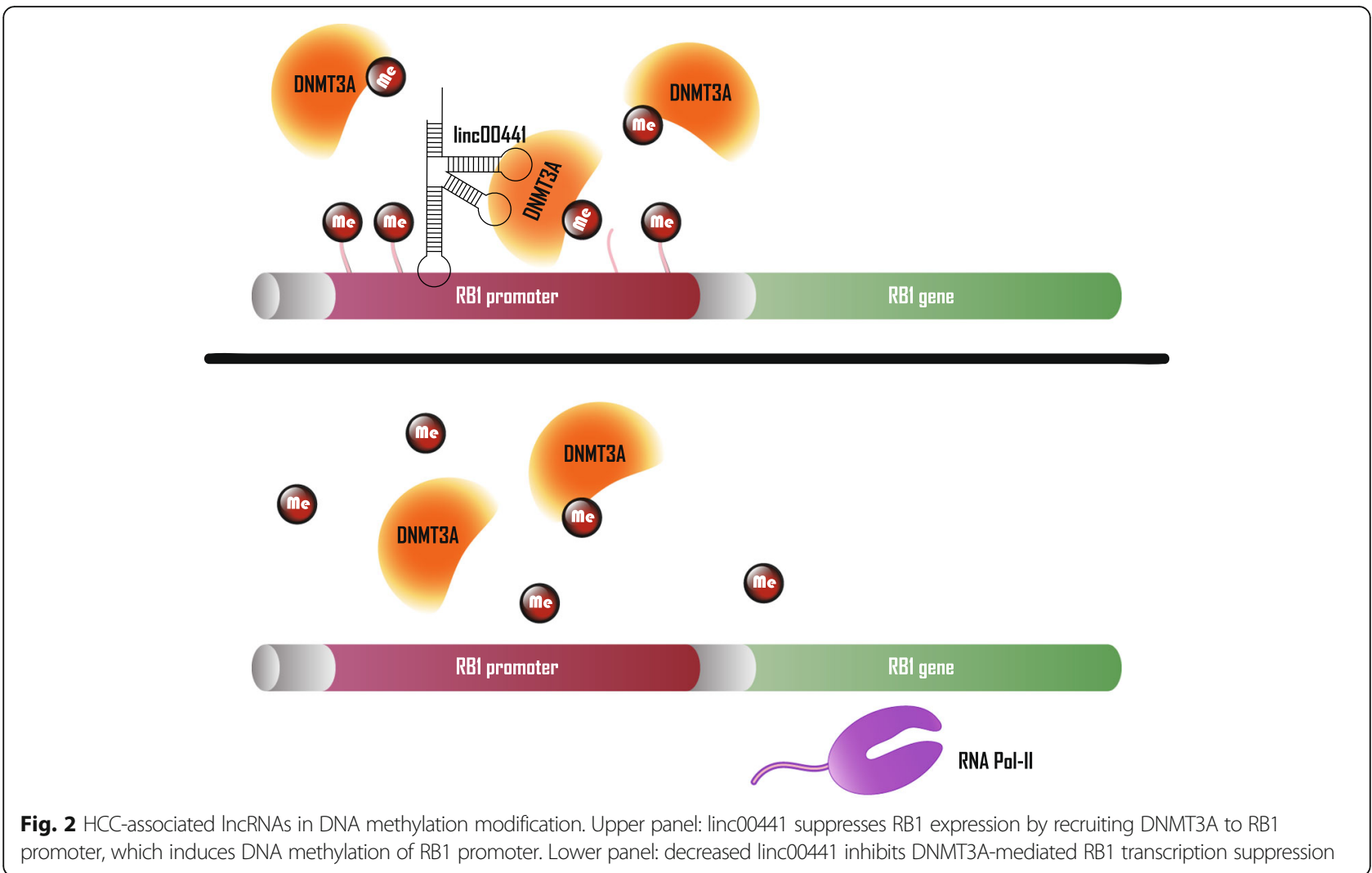

of p21 and BCL2-associated X protein (Fig. 3b) [54]. Conversely, HCC-associated lncRNAs can also sequester transcription factors to allow some target gene release from transcription inhibition. For example, a cis-positive feedback loop exists for the tumor suppressor molecules lncRNA-NEF and forkhead box A2 (FOXA2). LncRNA-NEF is mainly distributed in the cytoplasm, while $\beta$-catenin binds the FOXA2 promoter region and inhibits FOXA2 transcription in the nucleus. The direct interaction of lncRNA-NEF with $\beta$-catenin increases the cytoplasmic abundance of $\beta$-catenin. At the same time, Glycogen synthase kinase 3 beta (GSK3 $\beta$ ) also binds to, and increases the inhibitory phosphorylation of, $\beta$-catenin. Ultimately, lncRNA-NEF reduces nuclear levels of $\beta$-catenin, thereby increasing expression of FOXA2, which further promotes its transcription by binding IncRNA-NEF promoter [55].

Additionally, lncRNAs have been discovered that regulate gene expression by binding directly to the DNA element; that is, without interacting with transcription factors. A typical example of this in HCC is a lncRNA termed lncRNA downregulated in liver cancer stem cells (lnc-DILC). In contrast to lncSox4, down-regulated lnc-DILC in HCC inhibits STAT3 levels and suppresses liver cancer stem cell expansion. Using Basic Local Alignment Search Tool, Wang et al. identified a putative lnc-DILC complementary binding locus in the IL-6 promoter and verified that lnc-DILC inhibits NF-кB-mediated IL-6 transcription (Fig. 3c). Ultimately, lnc-DILC abolishes IL-6/JAK2/STAT3 autocrine signaling, and down-regulated lnc-DILC indicates poorer HCC prognosis [56]. Also, IncCAMTA1 plays a role in liver CSC expansion through similar mechanisms. As a transcript with higher expression levels in HCC and liver CSCs, lncCAMTA1 binds the calmodulin binding transcription activator 1 (CAMTA1) promoter and induces a suppressive chromatin structure, which leads to decreased CAMTA1 transcription. Subsequently reduced expression of CAMTA1 inhibits HCC cell proliferation and liver CSC-like properties [57]. Finally, TNF- $\alpha$ and IL-6 can stimulate expression of LINC000607, which represses NF-kB p65 transcription by binding to the NF- $\mathrm{kB}$ p 65 promoter region, eventually causing apoptosis due to elevated p53 expression [58]. In brief, HCC-associated IncRNAs can reduce transcription factors-DNA complexes by sequestering one of them, thereby inhibiting the downstream effects (Fig. 3b, c).

\section{HCC-associated IncRNAs in mRNA post- transcriptional regulation}

Transcripts originating from various protein-coding genes in the nucleus require a series of post-transcriptional steps to generate mature RNAs before translation, including: 5 '-end capping, alternative 


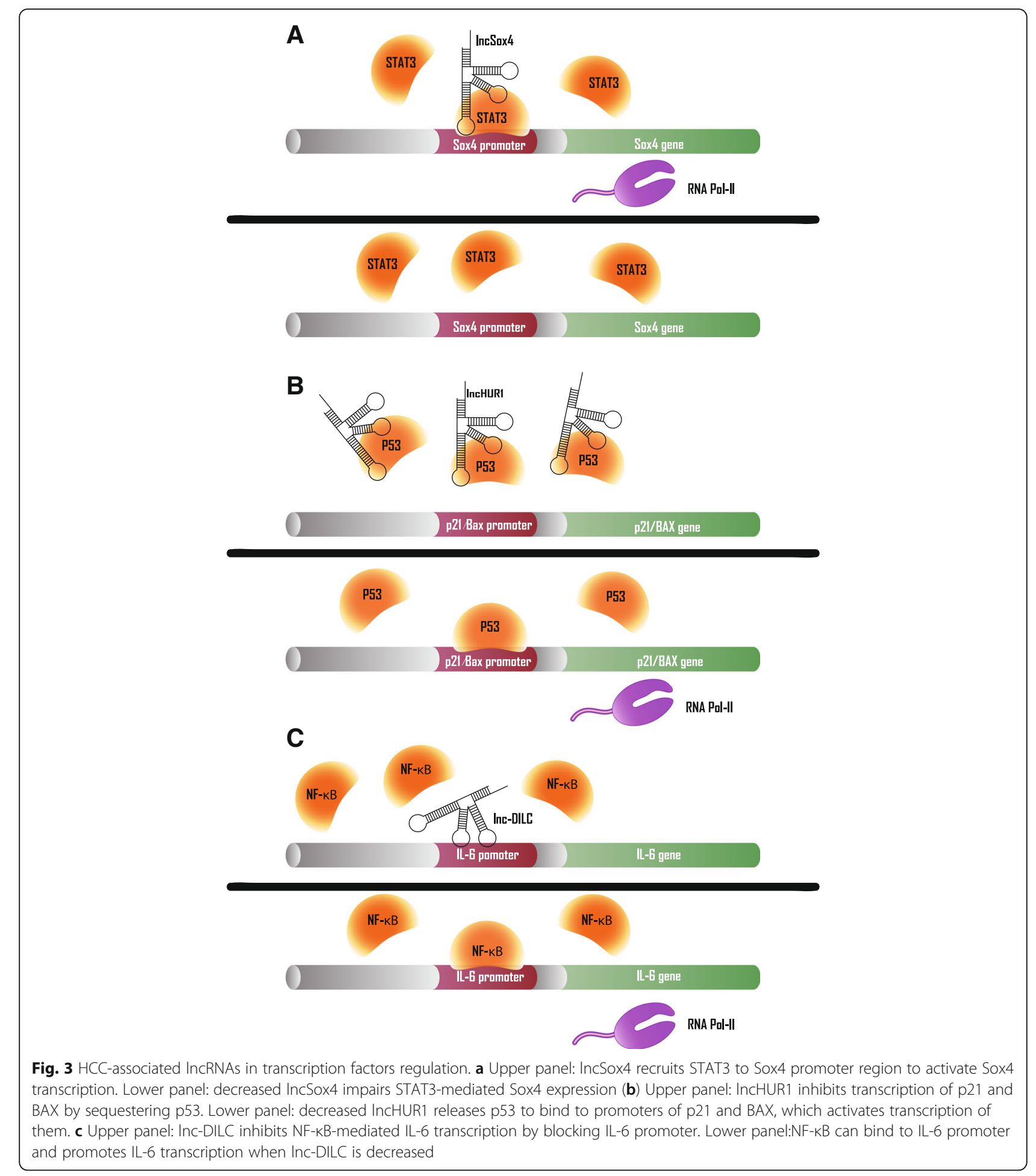

splicing and 3 '-end cleavage/polyadenylation. Then, mRNAs are transported to cytoplasm by RNA-binding proteins and mRNA export receptors. Once in the cytoplasm, mRNAs are translated into proteins or targeted for decay [59]. HCC-associated lncRNAs regulate mature mRNA expression by directly binding to complementary sequences on target mRNA or miRNAs, thereby decreasing protein expression levels. Similarly, miRNAs can bind to mRNA 3' - Untranslated Region (UTR) and assemble into miRNA-induced silencing complexes with Argonaute family proteins to silence gene expression [60]. In general, the more complementary the seed region and mRNA 
target sequence, the stronger the corresponding protein expression changes [61]. LncRNAs that contain the same miRNA response elements as mRNAs can promote mRNA translation by ceRNA, also known as "miRNA sponges" [33]. In recent years, as bioinformatics tools have advanced, knowledge of the sequence complementarity between lncRNA and mRNA is more accessible. Thus, ceRNA has become a more pervasive molecular mechanism of HCC-associated lncRNAs. The most representative example of this is lncRNA-PXN-AS1. Muscleblind like splicing regulator 3 (MBNL3) acts as an oncofetal splicing factor to increase the stability of paxillin (PXN) mRNA by alternative splicing of PXN antisense transcript 1 (PXN-AS1). Specifically, overexpression of MBNL3 stimulates exon 4 inclusion of PXN-AS1, which raises PXN-AS1-L levels and lowers PXN-AS1-S levels. Compared to PXN-AS1-S, PXN-AS1-L's unique exon 4 binds to the 3'-UTR region of PXN mRNA, which blocks miR-24 binding to this region and reduces degradation of PXN mRNA (Fig. 4a) [62]. Similarly, DANCR protects $\beta$-catenin from miR-214- or miR-320a-mediated degradation by binding to its mRNA 3'-UTR [63]. Interestingly, portal vein tumor thrombus-associated lncRNA ICAM-1Related Noncoding RNA (ICR) and Intercellular Adhesion Molecule 1 (ICAM-1) mRNA originated from partially overlapping locations in different strands of chromosome 19 , resulting in a complementary interaction between their respective, approximately $800 \mathrm{bp}$, regions at the $3^{\prime}$ end. These two transcripts are positively correlated in HCC, and ICR enhances the ICAM-1 mRNA stability by forming an RNA duplex with it, which may be due to blocking the binding site of certain miRNAs at the 3'-UTR [64].

In addition, some HCC-associated lncRNA-mRNA binding sites do not overlap with miRNA putative binding sites, but still affect the stability of the corresponding mRNAs. Hepatocellular Carcinoma Up-regulated Long Non-coding RNA (HULC) and Clock circadian regulator (CLOCK) mRNA 5'-UTR have a complementary base paring region, and the results of luciferase reporter gene assays suggest that HULC can improve the stability of CLOCK mRNA (Fig. 4b). Thereby, HULC disturbs circadian rhythm of HCC and accelerates hepatocarcinogenesis [65]. In contrast, lncARSR has been reported to promote the degradation of Phosphatase and tensin homolog (PTEN) mRNA by IncRNA-mRNA interaction in HCC, though the specific mechanism needs further characterization [66].

In addition to the above mechanism, to stabilize IL-11 mRNA and subsequently activate IL-11/STAT3 signaling, lncRNA-ATB can also indirectly improve mRNA stability and protein levels through the "miRNA sponge" model. Based on the TargetScan prediction algorithm, lncRNA-ATB was found to have three miR-200s target binding sites in a short span. And it was confirmed by quantitative real-time PCR that lncRNA-ATB and miR-200 s have approximately 100 and 200 copies per cell in SMMC-7721 cells, respectively. These conditions meet ceRNA mechanism criteria. Finally, it was verified by luciferase reporter gene assays and MS2-RNA immunoprecipitation that IncRNA-ATB sequesters miR-200s, thereby increasing the expression level of zinc finger E-box binding homeobox 1/2 (ZEB1/2) to induce epithelial-mesenchymal transition (Fig. 4c) [67]. This pattern is the most widely studied molecular mechanism in HCC-associated lncRNAs, revealing the ubiquitous IncRNA-miRNA-mRNA axis in HCC (Additional file 1: Table S1).

The above-mentioned mRNA post-transcriptional regulation has an obvious cellular compartment context. A study of two lncRNAs, IncRNA-UFC1 and MIR22 host gene (MIR22HG), supports the significant impact of subcellular location of some molecules on certain biological processes, especially mRNA post-transcriptional regulation. For instance, HuR (also known as ELAV like RNA binding protein 1) is a mRNA stabilizing protein for which its deregulated nucleus: cytoplasm ratio leads to tumor initiation and progression [68]. Interestingly, the interaction of $\mathrm{HuR}$ and Adenylate-uridylate-rich elements in the 3' UTR can enhance mRNA stability. $\beta$-catenin mRNA is one such example [69]. LncRNA-UFC1 acts as an HCC promoter by increasing cytoplasmic HuR levels, which results in more stable $\beta$-catenin mRNA [70]. Conversely, MIR22HG, a down-regulated lncRNA in HCC, competitively binds HuR with $\beta$-catenin and increases the nuclear fraction of HuR (Fig. 4d). That is to say, MIR22HG reduces $\beta$-catenin level by altering subcellular location of its mRNA stabilizing protein HUR [71]. Therefore, in addition to epigenetic modulation and transcription factor regulation, mRNA post-transcriptional regulation is another subcellular, location-dependent lncRNA mode of mechanism of action in HCC.

\section{HCC-associated IncRNAs in protein post- translational regulation and protein complex modulation}

HCC-associated lncRNAs regulate protein post-translationally via several molecular patterns. In protein degradation, lncRNAs promote or inhibit ubiquitination of proteins, which subsequently affects ubiquitin-proteasome-mediated protein degradation. LncRNAs can also modify proteins, represented by protein phosphorylation to have varying effects on their functions. Moreover, proteins often bind to each other to form protein complexes, and some HCC-associated lncRNAs transform the composition of subunits in various complexes. In these ways, HCC-associated lncRNAs affect protein post-translationallyand regulate the assembly of multiprotein complex . 

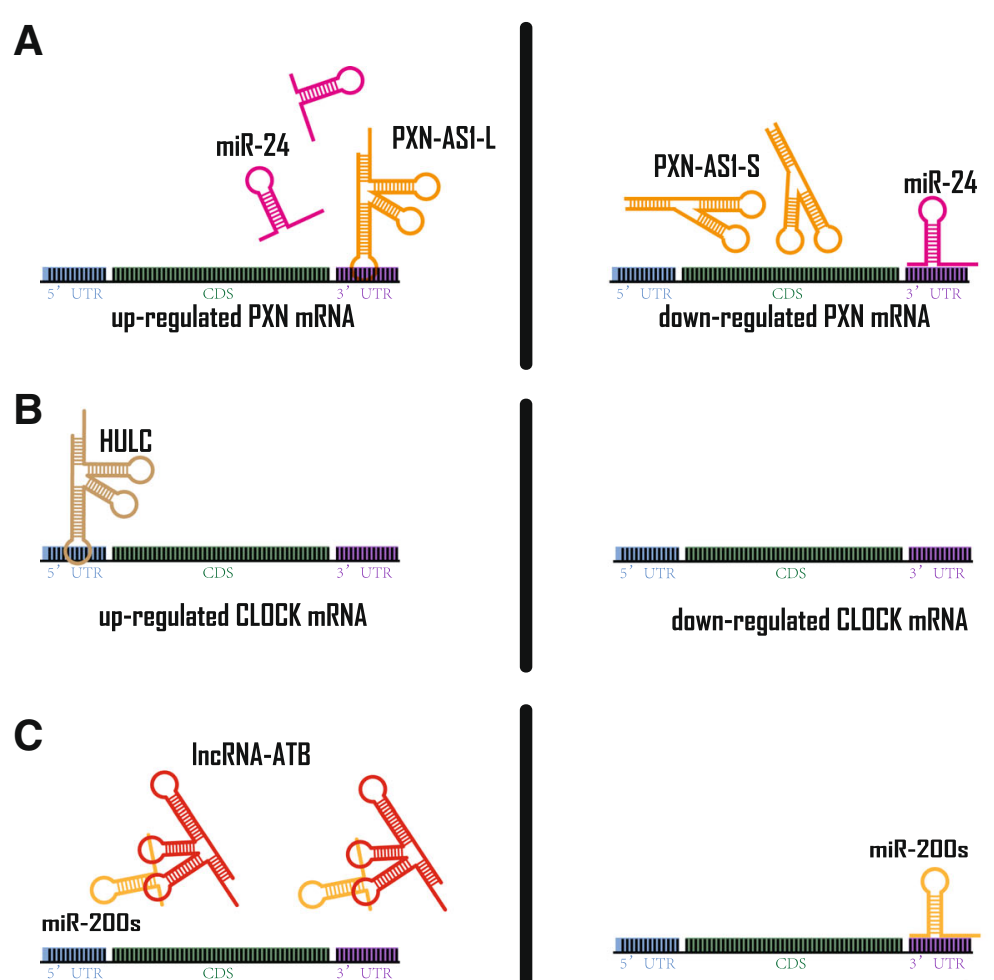

up-regulated ZEBI/2 mRNA
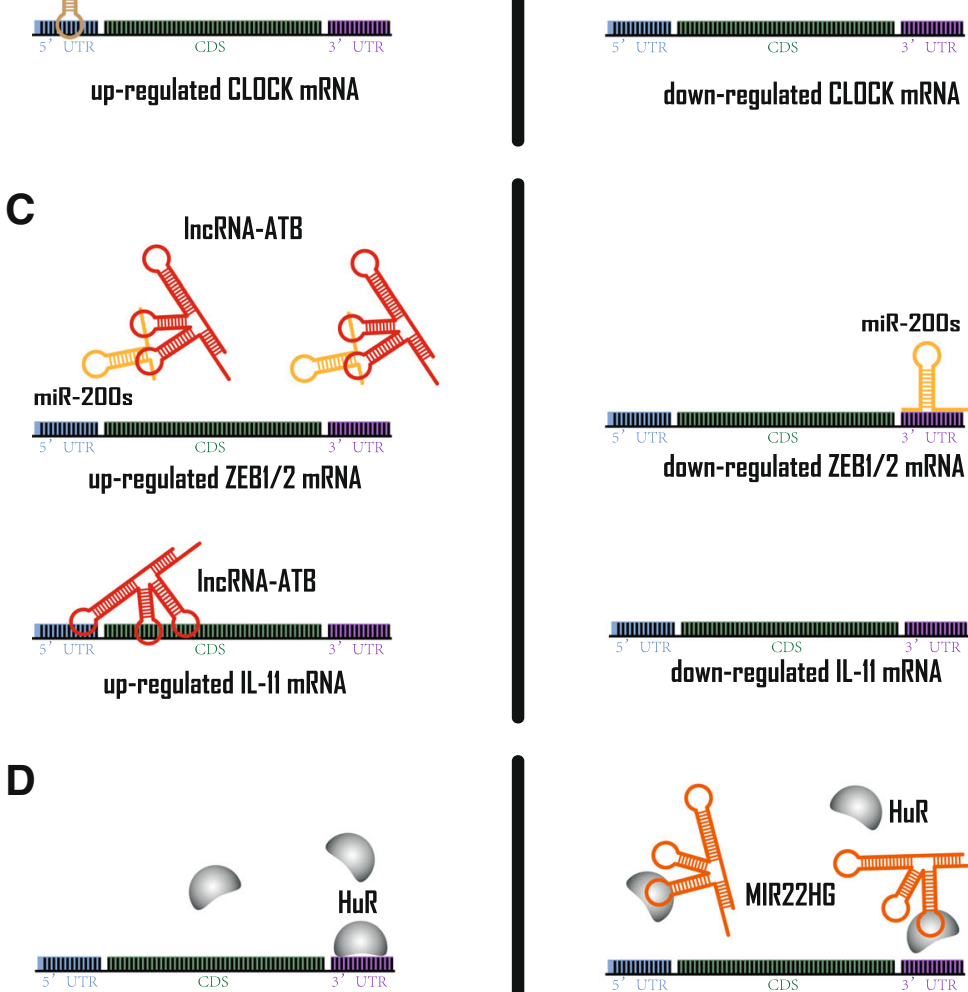

up-regulated $\beta$-catenin mRNA
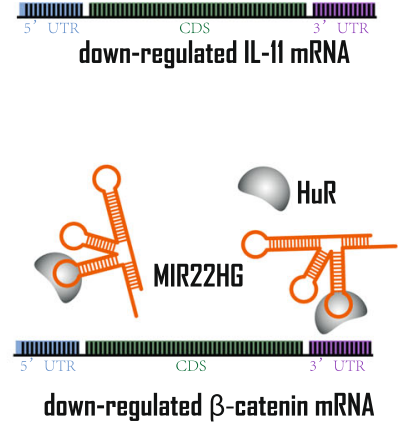

Fig. 4 HCC-associated IncRNAs in mRNA post-transcriptional regulation. a Left panel: PXN-AS1-L can sequester miR-24 with its exon4, thereby increasing PXN mRNA stability. Right panel: PXN-AS1-S does not consist of exon4, which releases miR-24 to bind to PXN mRNA 3'UTR. $\mathbf{b}$ Left panel: HULC can increase CLOCK mRNA stability by binding to its 5'UTR. Right panel: decreased HULC expression impairs CLOCK expression. c Left panel: IncRNA-ATB can increase ZEB1/2 mRNA stability by sequestering miR-200 s. Also, IncRNA-ATB can increase IL-11 mRNA stability by binding with it. Right panel: decreased IncRNA-ATB expression impairs ZEB1/2 and IL-11 expression. $\mathbf{d}$ Left panel: HuR can stabilize $\beta$-catenin by binding to its 3'UTR. Right panel: MIR22HG can down-regulate $\beta$-catenin mRNA by sequestering $\beta$-catenin mRNA

\section{Protein degradation}

There are multiple pathways for protein degradation in humans, which are generally classified into (1) degradation of dietary proteins, (2) degradation of extracellular proteins, and (3) degradation of intracellular proteins. Among them, the ubiquitin proteasome system (UPS) is a highly-specific cellular mode of protein degradation that plays a key role in maintaining protein quality and controlling cellular processes [72]. HCC-associated lncRNAs generally affect protein degradation through UPS, thereby regulating a series of signaling pathways.

26S proteasome recognizes ubiquitin-conjugated proteins and degrades them into small peptides. The process of ubiquitination requires E1 (ubiquitin-activating enzyme), E2 (ubiquitin-conjugating enzyme), and a substrate-specific E3 (ubiquitin-protein ligase) [72]. The 
interaction between E3 ligase and its target protein can be blocked by lncRNAs through sequestration. The direct interaction of E3 ligase Carboxy-Terminus of Hsc70 Interacting Protein (CHIP) with arginine methyltransferase 5 (PRMT5) prompted proteasomal degradation of PRMT5. LINC01138 was found to increase the protein level of PRMT5, but had no effect on the level of PRMT5 mRNA. Mechanistically, LINC01138 withholding PRMT5 results in significant inhibition of the association between CHIP and PRMT5 (Fig. 5a). Gene set enrichment analysis shows that LINC01138 and PRMT5 affect highly similar downstream signaling pathways, which may demonstrate that PRMT5 is a mediator of LINC01138's oncogenic role [73]. Furthermore, lnc-epidermal growth factor receptor (EGFR) similarly enhances EGFR stability, thereby increasing Treg cell differentiation and promoting the immunosuppressive state of HCC. More specifically, the domain (1001-1051 amino acids) of EGFR is exposed in the cytoplasm and is responsible for the physical interaction with lnc-EGFR, where Tyr1045 is the docking site of E3 ligase casitas B-lineage lymphoma (c-CBL). Collectively, lnc-EGFR inhibits the ubiquitination and degradation of subsequent EGFR by blocking the binding site of E3 ligase c-CBL, thereby promoting HCC progression [74].

In addition to sequestering, HCC-associated lncRNAs can also act as a scaffold to bridge the interaction between E3 ligase and its target protein to promote protein degradation. The DDX5 mentioned above directs the HOTAIR-PRC2 complex to epigenetically inhibit the transcription of specific genes. The human cancer stem cell marker EpCAM and pluripotency genes Nanog homebox, Oct4 (also known as POU class 5 homeobox 1) and Sox 2 do not match HOTAIR's role as an oncogenic factor [31]. Zhang et al. found that in the presence of $\mathrm{HBx}$, the E3 ligase Mex3b had more affinity for the PRC2 subunit SUZ12 and HOTAIR, whereas the interaction between DDX5 and these two molecules was reduced. As a replacement, Mex3b ubiquitinates SUZ12 and induces its degradation (Fig. 5b). HOTAIR acts as a molecular scaffold in both epigenetic regulation and ubiquitination, selectively affects gene expression, and drives $\mathrm{HBV}$-induced liver tumors with $\mathrm{HBx}$ [20]. Conversely, HCC-associated lncRNAs can also bind deubiquitinase and target proteins to inhibit proteolysis. For example, one of the pathways that oncogenic lncRNA LNC473 regulates links survivin and deubiquitinase ubiquitin specific peptidase 9 (USP9X) to suppress the ubiquitination level of survivin and increase survivin expression [75].

However, the effect of IncRNA on UPS-mediated protein degradation is not limited to the ubiquitination of target proteins. The lnc-b-Catm is a lncRNA that mediates protein methylation, which is identified by comparing CD13+ CD133+ cells with CD13-CD133cells. Lnc-b-Catm acts as a scaffold to enhance the interaction between EZH 2 and $\beta$-catenin, thus causing EZH2 to methylate $\beta$-catenin at K49 (Fig. 6c). This methylation then inhibits $\beta$-catenin's phosphorylation and subsequent ubiquitination, which inhibits degradation of $\beta$-catenin [76]. Furthermore, some HCC-associated lncRNAs bind proteins to increase their stability and expression levels, but understanding how the ubiquitination levels of these molecules requires further investigation $[24,25,77]$.

\section{Protein phosphorylation}

Similar to ubiquitination, HCC-associated lncRNAs also regulate protein phosphorylation primarily through two molecular modes of interaction: scaffolding and sequestering. Tumor suppressorlong noncoding RNA on chromosome 8p12 (TSLNC8) regulates phosphorylation of T705 and S727 on STAT3 by a relatively specific manner of sequestration. In short, lncRNAs interact with one of the two molecules, thereby inhibiting the interaction between them. Intriguingly, the right arm of TSLNC8 can be combined with transketolase (TKT) or STAT3 to allow the two molecules to compete with each other. That is, TSLNC8, TKT and STAT3 can combine with each other, but the presence of TSLNC8 reduces the interaction between TKT and STAT3. Eventually, overexpression of TLSNC8 results in a decrease in STAT3 Y705 phosphorylation and an increase in S727 phosphorylation, which can significantly attenuate the oncogenic ability of STAT3 (Fig. 6a) [26]. Unlike TSLNC8, HULC can simultaneously bind Y-box binding protein 1 (YB-1) and extracellular signal-regulated kinase (ERK) to promote phosphorylation of YB-1 by ERK (Fig. 6b). Phosphorylation of YB-1 results in the release of some mRNAs, which ultimately accelerates the translation of these mRNAs. The resulting increase in cyclin D1 and cyclin E1 promotes G1/S transition. This represents another mechanism by which HCC can develop due to HULC action [27]. HCC associated long non-coding RNA (HANR) appears to detain the GSK3B-interacting protein (GSKIP), which hinders GSKIP-mediated GSK-3 $\beta$ phosphorylation. GSK-3 $\beta$ promotes the phosphorylation of $\beta$-catenin and causes it to be hydrolyzed by the proteasome pathway. Therefore, HANR is also an HCC oncogenic factor that affects protein phosphorylation $[78,79]$.

Furthermore, Ding et al. proposed that HNF1A antisense RNA 1 (HNF1A-AS1) modulates the phosphatase activity of $\mathrm{SH} 2$-containing protein tyrosine phosphatase-1 (SHP-1) by binding with its C-terminal. Nevertheless, how such interaction enhances the enzymatic activity of SHP-1 remains to be characterized [80]. 

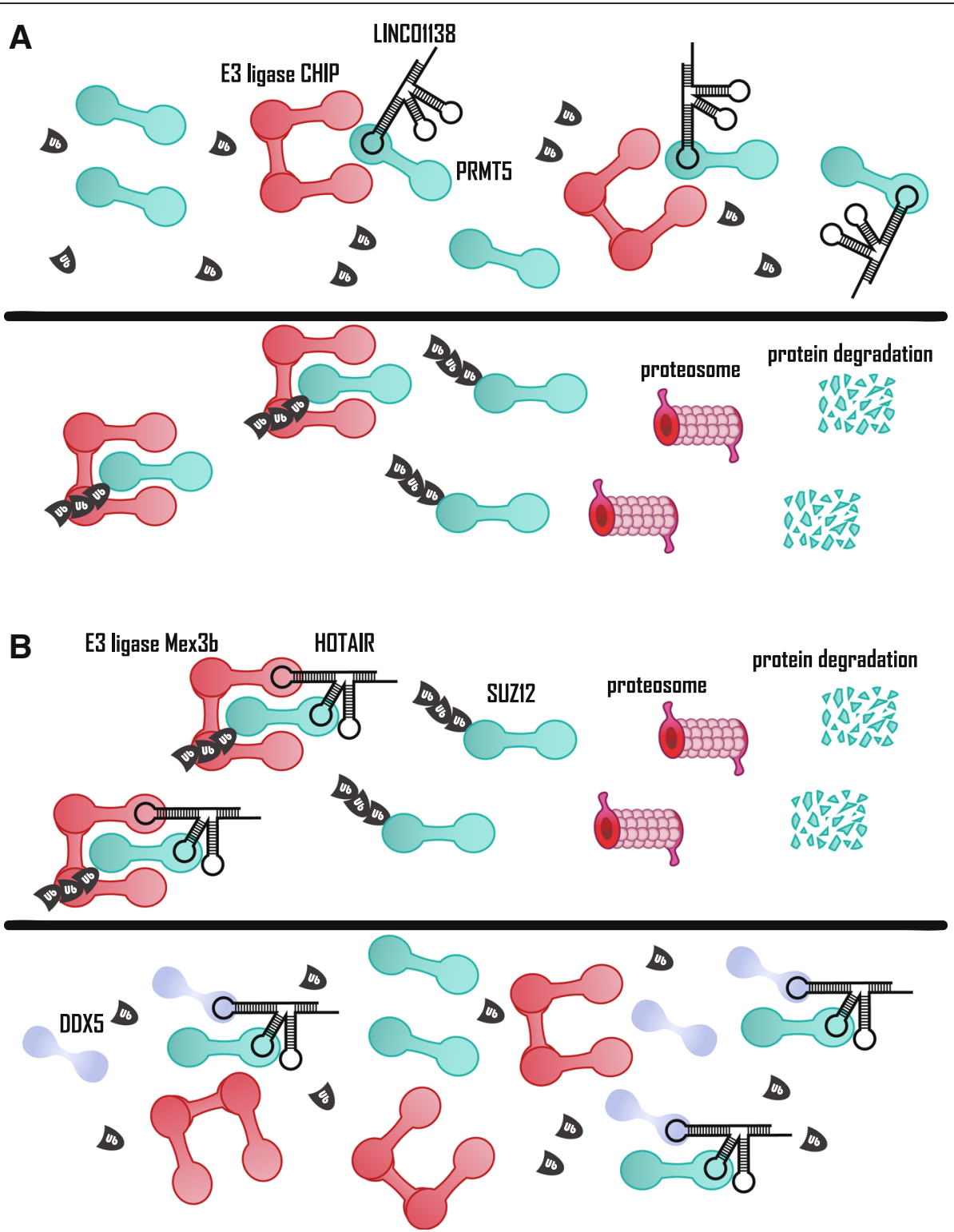

Fig. 5 HCC-associated IncRNAs in protein degradation. a Upper panel: LINC01138 blocks E3 ligase CHIP-mediated ubiquitination of PRMT5 by sequestering PRMT5. Lower panel: decreased LINC01138 leads to release of PRMT5, which leads to E3 ligase CHIP-mediated ubiquitination of PRMT5 and its degradation. b Upper panel: HOTAIR acts as scaffold to bridge E3 ligase Mex3b and SUZ12, a core subunit of PRC2 complex, which leads to ubiquitination and degradation of SUZ12. Lower panel: DDX5 inhibits E3 ligase Mex3b-mediated SUZ12 protein degradation by displacing the Mex3b from HOTAIR

\section{Protein complex modulation}

The molecular mode of interaction of HCC-associated lncRNAs in protein complex modulation is the same as in the first two sections; that is, by sequestering and as a scaffold. First, IncBRM indirectly facilitates the assembly of a subunit into the BRG1-associated factor (BAF) complex by splitting another subunit, which is a type of subunit switch. LncBRM, another lncRNA derived by comparing CD13 + CD133+ cells and CD13-CD133-cells transcriptome, binds to Brahma (BRM), resulting in a decrease in BRM-embedded BAF complex. Correspondingly, the BRG1 act as a substitute for BRM to form the BRG1-embedded BAF complex. The difference between the two complexes is that BRG1 can bind to transcription factor Kruppel-like factor 4 (KLF4), while there is no interaction between BRM and KLF4. The BRG1-embedded BAF complex is directed by KLF4 to the Yes-associated protein 1 (YAP1) promoter, which facilitates YAP1 transcription (Fig. 7). Therefore, the increase in IncBRM ultimately promotes the LCSC property of cells by activating the BAF/YAP1 pathway [81]. 
A

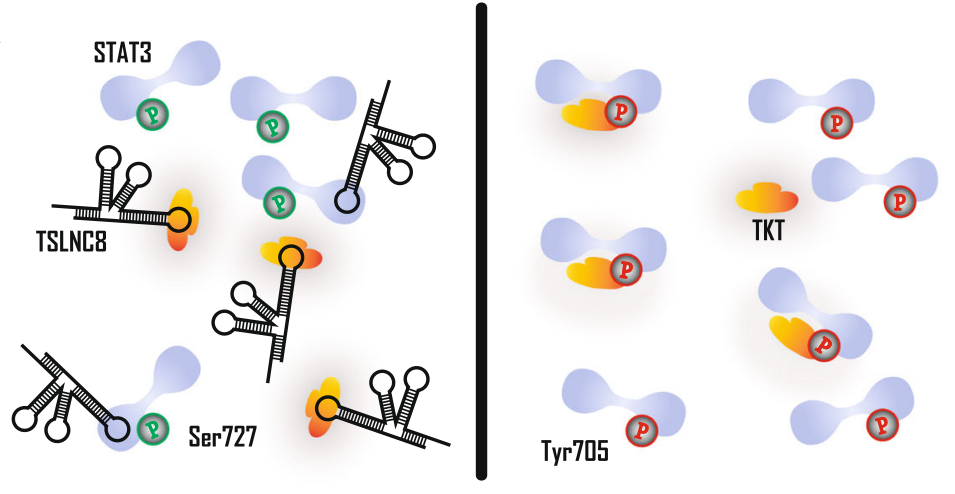

B
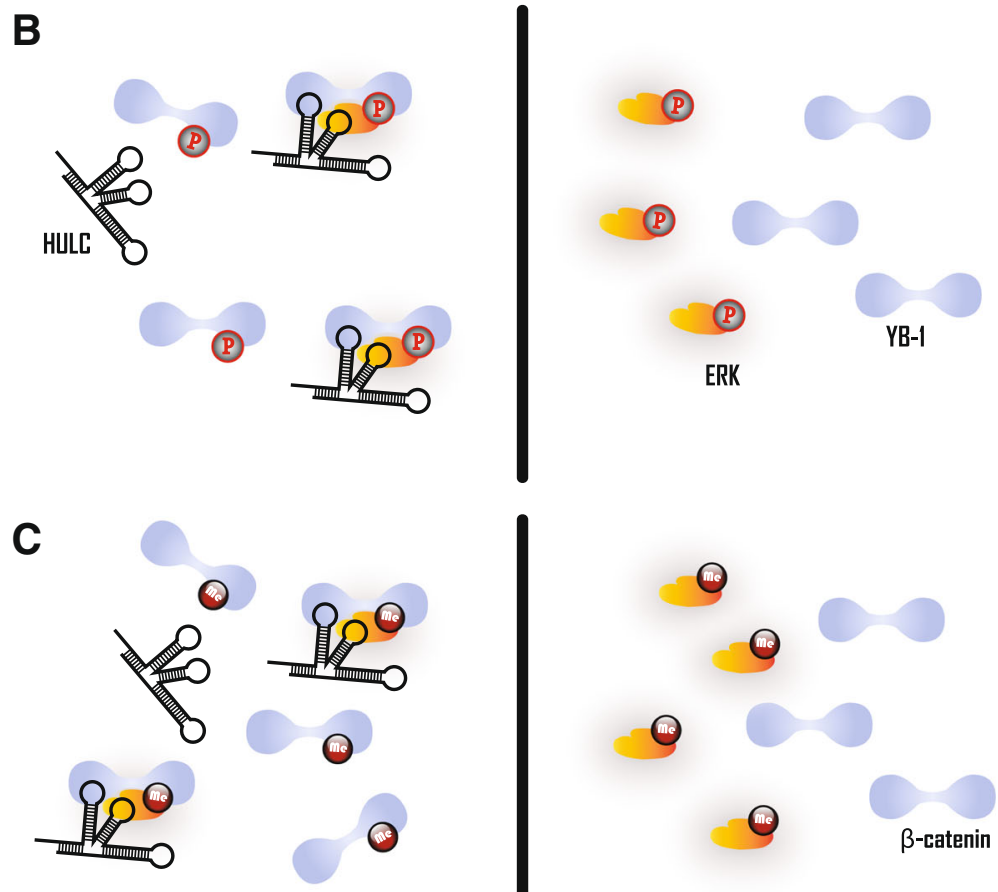

Inc- $\beta$-Latm

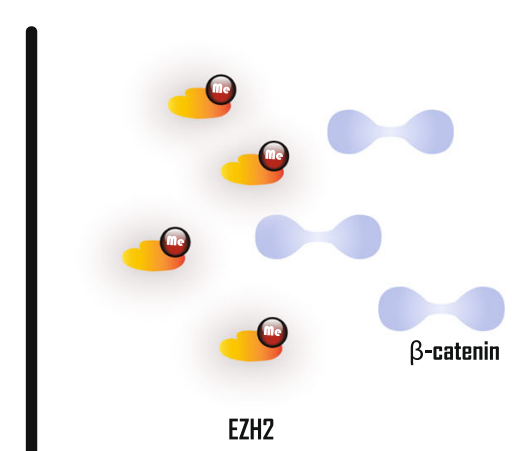

Fig. 6 HCC-associated IncRNAs in protein modification. a Left panel: TSLNC8 disrupts TKT-mediated STAT phosphorylation by sequestering STAT3 or TKT, which induces Ser727 phosphorylation and Tyr705 dephosphorylation in STAT3. Right panel: decreased TSLNC8 promotes the interaction between STAT3 and TKT, which induces TKT-mediated Ser727 dephosphorylation and Tyr705 phosphorylation in STAT3. b Left panel: HULC promotes ERK mediated YB-1 phosphorylation by acting as scaffold to bridge ERK and YB-1. Right panel: decreased HULC impairs ERK mediated YB-1 phosphorylation. $\mathbf{c}$ Left panel: Inc- $\beta$-Catm promotes ERK mediated $\beta$-catenin methylation by acting as scaffold to bridge EZH 2 and $\beta$-catenin. Right panel: decreased Inc- $\beta$-Catm impairs EZH2 mediated $\beta$-catenin methylation

Another example is lnc-Tim3-mediated HCC CD8 T lymphocyte exhaustion, which compromises HCC anti-tumor immunity. Briefly, Bat3 can simultaneously bind the C-terminal intracellular domain of Tim-3 (also known as hepatitis A virus cellular receptor 2) and Lck (also known as Src family tyrosine kinase). The formation of this complex activates $\mathrm{T}$ cell signaling (ZAP70/AP-1/NFAT1) and suppresses CD8 $\mathrm{T}$ cell exhaustion. However, lnc-Tim3 interferes with this process by binding the $\mathrm{C}$-terminal intracellular domain of Tim-3, thereby releasing BAT3 from Tim3. BAT3 is then free to form a complex with p300 and recruit p300 into the nucleus, which exacerbates CD8 T cell exhaustion [82]. A similar phenomenon also occurs in the Wnt/ $\beta$-catenin signaling pathway regulated by Linc00210 in TIC. The regulation of the $\mathrm{Wnt} / \beta$-catenin signaling pathway is dependent on the alternative assembling of proteins as subunits in various complexes [79]. Linc00210 binds to catenin beta interacting protein 1 (CTNNBIP1) and impairs the interaction between $\beta$-catenin and CTNNBIP1. CTNNBIP1 acts as a Wnt/ $\beta$-catenin signaling pathway suppressor that can detain and prevent $\beta$-catenin from binding to TCF/LEF components. Without $\beta$-catenin, 


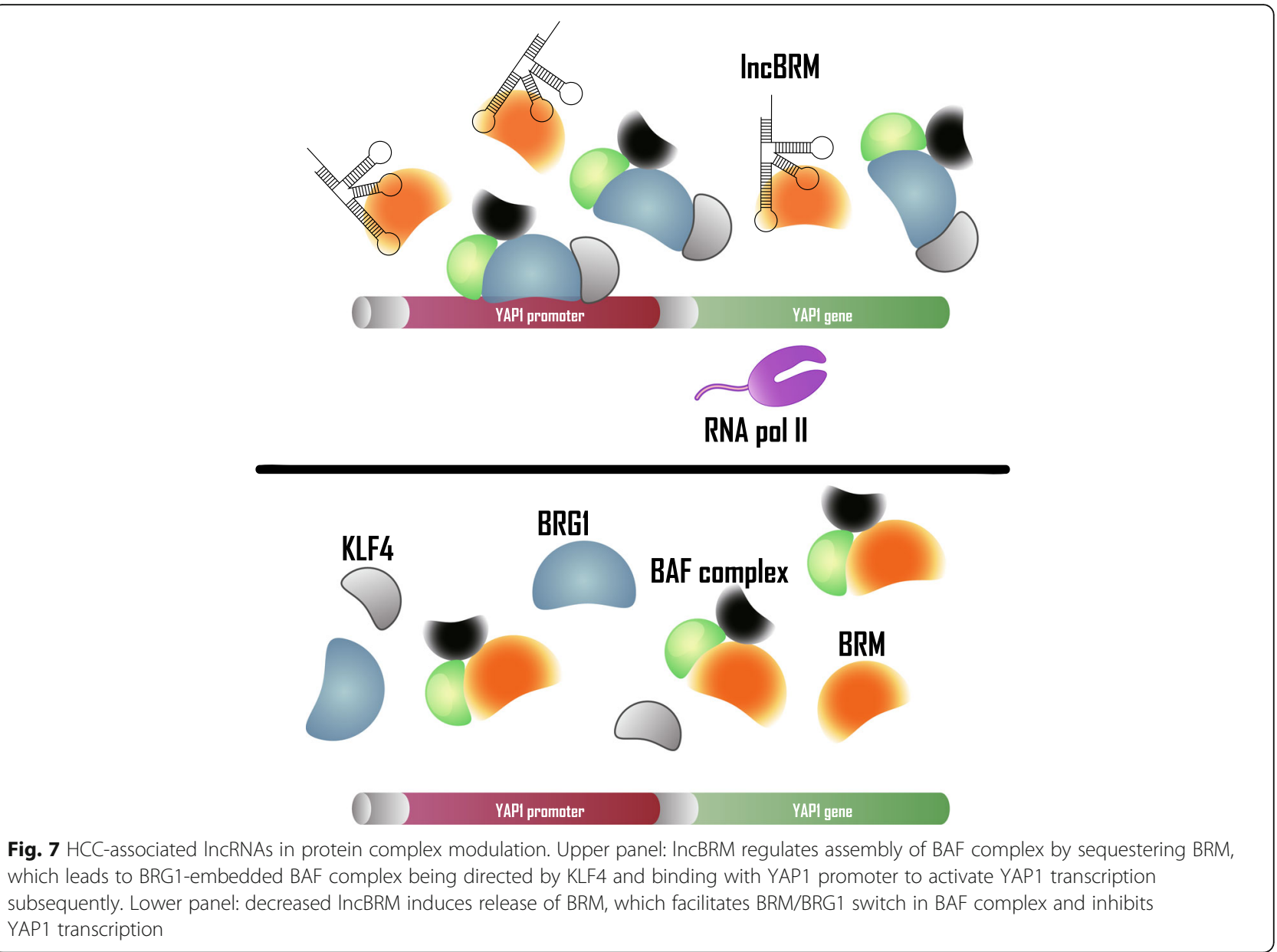

TCF/LEF components act as transcription repressors. $\beta$-catenin is an activator of TCF/LEF components to release the repressed gene. Through this series of consecutive steps, Linc 00210 became an HCC-related oncogene by means of protein complex modulation [83]. In addition, as described above, GSK-3 $\beta$ can inhibit $\beta$-catenin by phosphorylation, and the interaction between GSK-3 $\beta$ and Annexin A2 is enhanced by scaffolding of lncRNA-MUF. Eventually, $\beta$-catenin escapes inhibition by GSK-3 $\beta$, and is thus able to accumulate and translocate to nucleus [28].

Although different in biological processes, HCC-associated $\operatorname{lncRNAs}$ regulate protein degradation, protein modification, and protein complexes primarily by affecting interactions between other molecules. In short, HCC-associated lncRNAs affect these biological processes through two molecular interaction modes: sequester and scaffold. However, HCC-associated lncRNAs may not be just a molecular interaction partner, although this assumption requires stronger evidence to support.

\section{Conclusion and perspective}

As the largest class of transcripts in humans, lncRNAs can affect almost any realm of cellular biology. In order to fully understand the cellular mechanisms underlying the development of diseases such as HCC, it is necessary to understand lncRNA function. To our knowledge, no comprehensive model yet exists for the classification of HCC-associated lncRNAs. There is a considerable contribution by lncRNAs to the "transcriptional noise" that impacts the central dogma of the "DNA-RNA-protein" axis. Therefore, this review focuses on transcription and translation, classifies lncRNAs according to biological processes, and further subdivides them by their most common modes of molecular interaction in HCC. This newly streamlined classification method provides a more approachable system by which to study and discuss lncRNAs in HCC. We must note, however, there are still exceptional HCC-associated lncRNAs that are not suitable for this classification method, such as MVIH and Dreh [84, 85]. Moreover, some lncRNAs have been characterized to regulate gene expression via other mechanisms under other physiological and pathological conditions. For instance, lncRNA termed functional intergenic repeating RNA element (Firre), that promotes cross-chromosomal interactions as a trans-acting platform in several cell lines [86], while Colorectal Cancer 
Associated Transcript 1-L facilitates long-range interactions between MYC promoter and its enhancers in cis in colorectal cancer [87]. And lncRNAs generated from Alu SINE elements can repress the transcription machinery directly by binding to RNA polymerase II (Pol II) during heat shock [88]. Given that lncRNAs comprise $68 \%$ of the human transcriptome, future research may reveal new categories or definitions of lncRNAs.

High-throughput sequencing technology, followed by functional studies, have helped to identify and elucidate the role of a large number of IncRNAs in HCC over the past decade. However, the vast majority of lncRNAs still need to be investigated. Given the worldwide impact on morbidity and mortality by HCC, it is important that future research focuses on IncRNAs. In addition, the mechanisms of aberrant gene expression caused by lncRNAs in HCC has been more explicitly studied (Additional file 1: Table S1). Interestingly, it has also been noted that prostate cancer-associated single nucleotide polymorphisms (SNPs) are mainly enriched in regulatory regions, some of which are located in lncRNAs and can affect the their functions [89]. A similar association may exist for HCC, and by integrating genome-wide association studies and transcriptome data, it may be possible to discover and elucidate the mechanisms of some IncRNA-related SNPs. This has been practiced in several studies of various cancers, including HCC [89-94]. In the near future, research on the role of SNP-mediated HCC-related lncRNAs should be rewarded $[95,96]$, as this progress may become the key to gaining a greater understanding of the development of HCC.

\section{Additional file}

Additional file 1: Table S1. Summary of HCC-associated IncRNAs. (DOCX $171 \mathrm{~kb})$

\footnotetext{
Abbreviations

BAF: BRG1-associated factor; BAX: BCL2-associated X protein; BRM: Brahma; CAMTA1: calmodulin binding transcription activator 1 ; C-CBL: casitas Blineage lymphoma; ceRNA: competing endogenous RNA; CHIP: CarboxyTerminus of Hsc70 Interacting Protein; CLOCK: Clock circadian regulator; CTNNBIP1: catenin beta interacting protein 1; DDX5: RNA Helicase DEAD Box Protein 5; DNMT1: DNA methyltransferase 1; DNMT3: DNA methyltransferase 3; EGFR: Epidermal growth factor receptor; EMT: Epithelial to mesenchymal; EpCAM: Epithelial cell adhesion molecule; ERK: Extracellular signal-regulated kinase; EZH2: Enhancer of zeste homolog 2; Firre: Functional intergenic repeating RNA element; FOXA2: Forkhead box A2; GIHCG: Gradually Increased During Hepatocarcinogenesis; GPC3-AS1: GPC3 antisense RNA 1; GSK3ß: Glycogen synthase kinase 3 beta; GSKIP: GSK3B-interacting protein; HANR: HCC associated long non-coding RNA; HBV: Hepatitis B virus; HCC: Hepatocellular carcinoma; HNF1a: HNF1 homeobox A; HNF1AAS1: HNF1A antisense RNA 1; HNF4a: Hepatocyte nuclear factor 4, alpha; HOTAIR: HOX transcript antisense RNA; HULC: Hepatocellular Carcinoma Upregulated Long Non-coding RNA; ICAM-1: Intercellular Adhesion Molecule 1; ICR: ICAM-1-Related Noncoding RNA; KLF4: Kruppel-like factor 4; IncDILC: IncRNA downregulated in liver cancer stem cells; IncRNAs: long noncoding RNAs; MBNL3: Muscleblind like splicing regulator 3; Mex3b: Mex-3 RNA-binding family member B; MIR22HG: MIR22 host gene;
}

miRNAs: microRNAs; Pol II: RNA polymerase II; PRC2: Polycomb repressive complex 2; PRMT5: Arginine methyltransferase 5; PTEN: Phosphatase and tensin homolog; PXN: Paxillin; PXN-AS1: PXN antisense transcript 1; RB1: Retinoblastoma gene 1; SHP-1: SH2-containing protein tyrosine phosphatase; SIX3: SIX homebox 3; SNPs: Single nucleotide polymorphisms; Sox4: Sex determining region Y-box 4; STAT3: Signal transducer and activator of transcription 3; SUZ12: Subunit suppressor of zeste 12 homolog; SWI/ SNF: SWItch/Sucrose Non-Fermentable; TCF7: Transcription factor 7; TIC: Tumor-initiating cells; TKT: Transketolase; TSLNC8: Tumor suppressor long noncoding RNA on chromosome 8p12; UPS: Ubiquitin proteasome system; USP9X: Ubiquitin specific peptidase 9; UTR: Untranslated Region; WD: Repeat domain 26; YAP1: Yes-associated protein 1; YB-1: Y-box binding protein 1; ZEB1/2: Zinc finger E-box binding homeobox 1/2

\section{Acknowledgements}

Not applicable.

\section{Funding}

This study was supported by the General Programs from the National Natural Science Foundation of China (No. 81472618 and 81670535), the National Science and Technology Major Project (No. 2017ZX10202202 and 2018ZX10301202), the Local Innovative and Research Teams Project of Guangdong Pearl River Talents Program (No. 2017BT01S131), the Innovative Research Team Project of Guangxi Province (no. 2017GXNSFGA198002), the Grant for Recruited Talents to Start Scientific Research from Nanfang Hospital, and the Distinguished Youth Scholars Incubation Project of Nanfang Hospital (No. 2017 J001) to De-Ke Jiangs.

\section{Availability of data and materials}

Data sharing not applicable to this article as no datasets were generated or analyzed during the current study.

\section{Conflict of interests}

There are no known conflicts of interest.

\section{Authors' contributions}

HM wrote the review article. BZ, LL, FY, CC, YJ, JH and DJ reviewed the manuscript. All authors read and approved the final manuscript.

Ethics approval and consent to participate

Not applicable.

Consent for publication

Not applicable.

\section{Competing interests}

The authors declare that they have no competing interests.

\section{Publisher's Note}

Springer Nature remains neutral with regard to jurisdictional claims in published maps and institutional affiliations.

\section{Author details \\ ${ }^{1}$ State Key Laboratory of Organ Failure Research, Guangdong Key Laboratory of Viral Hepatitis Research, Institute of Liver Diseases Research of Guangdong Province, Guangzhou, China. ${ }^{2}$ Department of Infectious Diseases and Hepatology Unit, Nanfang Hospital, Southern Medical University, Guangzhou 510515, China. ${ }^{3}$ Department of Medical Genetics, Second Military Medical University, Shanghai 200433, China. ${ }^{4}$ University of Illinois College of Medicine, Chicago, IL 60612, USA. ${ }^{5}$ Department of Public Health Sciences, University of Chicago, Chicago, IL 60637, USA.}

Received: 21 March 2019 Accepted: 7 May 2019

Published online: 16 May 2019

\section{References}

1. Bray F, Ferlay J, Soerjomataram I, et al. Global cancer statistics 2018: GLOBOCAN estimates of incidence and mortality worldwide for 36 cancers in 185 countries. CA Cancer J Clin. 2018;68:394-424.

2. Zucman-Rossi J, et al. Genetic Landscape and Biomarkers of Hepatocellular Carcinoma. Gastroenterology. 2015;149(5):1226-39 e4. 
3. Crick F. Central dogma of molecular biology. Nature. 1970;227(5258):561-3.

4. Lander $\mathrm{E}$, et al. Initial sequencing and analysis of the human genome. Nature. 2001;409(6822):860-921.

5. Liang $F$, et al. Gene index analysis of the human genome estimates approximately 120,000 genes. Nat Genet. 2000;25(2):239-40.

6. International Human Genome Sequencing C. Finishing the euchromatic sequence of the human genome. Nature. 2004;431:931-45.

7. Djebali S, et al. Landscape of transcription in human cells. Nature. 2012; 489(7414):101-8.

8. Consortium, E.P. An integrated encyclopedia of DNA elements in the human genome. Nature. 2012;489(7414):57-74

9. Struhl K. Transcriptional noise and the fidelity of initiation by RNA polymerase II. Nat Struct Mol Biol. 2007;14(2):103-5.

10. Pennisi E. Genomics. ENCODE project writes eulogy for junk DNA. Science. 2012;337(6099):1159-61.

11. lyer MK, et al. The landscape of long noncoding RNAs in the human transcriptome. Nat Genet. 2015:47(3):199-208.

12. Guttman $\mathrm{M}$, et al. Chromatin signature reveals over a thousand highly conserved large non-coding RNAs in mammals. Nature. 2009;458(7235):223-7.

13. Rinn JL, Chang HY. Genome regulation by long noncoding RNAs. Annu Rev Biochem. 2012;81:145-66.

14. Derrien T, et al. The GENCODE v7 catalog of human long noncoding RNAs: analysis of their gene structure, evolution, and expression. Genome Res. 2012;22(9):1775-89.

15. Kopp F, Mendell JT. Functional classification and experimental dissection of long noncoding RNAs. Cell. 2018;172(3):393-407.

16. Geisler S, Coller J. RNA in unexpected places: long non-coding RNA functions in diverse cellular contexts. Nat Rev Mol Cell Biol. 2013;14(11):699-712.

17. Quinn JJ, Chang HY. Unique features of long non-coding RNA biogenesis and function. Nat Rev Genet. 2016;17(1):47-62

18. Schmitt AM, Chang HY. Long noncoding RNAs in Cancer pathways. Cancer Cell. 2016;29(4):452-63.

19. Klingenberg $M$, et al. Non-coding RNA in hepatocellular carcinoma: mechanisms, biomarkers and therapeutic targets. J Hepatol. 2017;67(3):603-18.

20. Zhang $\mathrm{H}$, et al. RNA helicase DEAD box protein 5 regulates Polycomb repressive complex 2/Hox transcript antisense intergenic RNA function in hepatitis B virus infection and hepatocarcinogenesis. Hepatology. 2016;64(4):1033-48.

21. Li Z, et al. The LINC01138 drives malignancies via activating arginine methyltransferase 5 in hepatocellular carcinoma. Nat Commun. 2018;9(1): 1572.

22. Jiang $R$, et al. The long noncoding RNA Inc-EGFR stimulates T-regulatory cells differentiation thus promoting hepatocellular carcinoma immune evasion. Nat Commun. 2017;8:15129.

23. Ni W, et al. A novel IncRNA uc.134 represses hepatocellular carcinoma progression by inhibiting CUL4A-mediated ubiquitination of LATS1. J Hematol Oncol. 2017;10(1):91.

24. Zhou CC, et al. Systemic genome screening identifies the outcome associated focal loss of long noncoding RNA PRAL in hepatocellular carcinoma. Hepatology. 2016;63(3):850-63.

25. Yang F, et al. Repression of the long noncoding RNA-LET by histone deacetylase 3 contributes to hypoxia-mediated metastasis. Mol Cell. 2013; 49(6):1083-96.

26. Zhang J, et al. Long noncoding RNA TSLNC8 is a tumor suppressor that inactivates the interleukin-6/STAT3 signaling pathway. Hepatology. 2018; 67(1):171-87.

27. Li D, et al. Long noncoding RNA HULC modulates the phosphorylation of YB-1 through serving as a scaffold of extracellular signal-regulated kinase and YB-1 to enhance hepatocarcinogenesis. Hepatology. 2017;65(5):1612-27.

28. Yan X, et al. Mesenchymal stem cells promote Hepatocarcinogenesis via IncRNAMUF interaction with ANXA2 and miR-34a. Cancer Res. 2017;77(23):6704-16.

29. Au SL, et al. Enhancer of zeste homolog 2 epigenetically silences multiple tumor suppressor microRNAs to promote liver cancer metastasis. Hepatology. 2012;56(2):622-31.

30. Khalil A, et al. Many human large intergenic noncoding RNAs associate with chromatin-modifying complexes and affect gene expression. Proc Natl Acad Sci U S A. 2009;106(28):11667-72.

31. Gupta RA, et al. Long non-coding RNA HOTAIR reprograms chromatin state to promote cancer metastasis. Nature. 2010;464(7291):1071-6.

32. Fu WM, et al. Hotair mediates hepatocarcinogenesis through suppressing miRNA-218 expression and activating P14 and P16 signaling. J Hepatol. 2015;63(4):886-95.
33. Salmena $L$, et al. A ceRNA hypothesis: the Rosetta stone of a hidden RNA language? Cell. 2011;146(3):353-8.

34. Jin $L$, et al. LncRNA GHET1 predicts poor prognosis in hepatocellular carcinoma and promotes cell proliferation by silencing KLF2. J Cell Physiol. 2018;233(6):4726-34

35. Wang C, et al. Long non-coding RNA HNF1A-AS1 promotes hepatocellular carcinoma cell proliferation by repressing NKD1 and P21 expression. Biomed Pharmacother. 2017;89:926-32.

36. Zeng $B$, et al. Upregulation of $L n C D Q$ is associated with poor prognosis and promotes tumor progression via epigenetic regulation of the EMT pathway in HCC. Cell Physiol Biochem. 2018;46(3):1122-33.

37. Wei C, et al. LncRNA SOX21-AS1 is associated with progression of hepatocellular carcinoma and predicts prognosis through epigenetically silencing p21. Biomed Pharmacother. 2018;104:137-44.

38. Zhou M, Zhang XY, Yu X. Overexpression of the long non-coding RNA SPRY4-IT1 promotes tumor cell proliferation and invasion by activating EZH2 in hepatocellular carcinoma. Biomed Pharmacother. 2017;85:348-54.

39. Huang MD, et al. Long non-coding RNA ANRIL is upregulated in hepatocellular carcinoma and regulates cell apoptosis by epigenetic silencing of KLF2. J Hematol Oncol. 2015;8:50.

40. Davidovich C, et al. Promiscuous RNA binding by Polycomb repressive complex 2. Nat Struct Mol Biol. 2013;20(11):1250-7.

41. Kretz M, Meister G. RNA binding of PRC2: promiscuous or well ordered? Mol Cell. 2014;55(2):157-8.

42. Battistelli $\mathrm{C}$, et al. The snail repressor recruits EZH2 to specific genomic sites through the enrollment of the IncRNA HOTAIR in epithelial-to-mesenchymal transition. Oncogene. 2017:36(7):942-55.

43. Yang Z, et al. Overexpression of long non-coding RNA HOTAIR predicts tumor recurrence in hepatocellular carcinoma patients following liver transplantation. Ann Surg Oncol. 2011;18(5):1243-50.

44. Li C, Chen Y. Targeting long non-coding RNAs in cancers: progress and prospects. Int J Biochem Cell Biol. 2013;45(8):1895-910.

45. Sui CJ, et al. Long noncoding RNA GIHCG promotes hepatocellular carcinoma progression through epigenetically regulating miR-200b/a/429. J Mol Med (Berl). 2016;94(11):1281-96.

46. Tang J, et al. Bidirectional transcription of Linc00441 and RB1 via H3K27 modification-dependent way promotes hepatocellular carcinoma. Cell Death Dis. 2017:8(3):e2675.

47. Zhang L, et al. Epigenetic activation of the MiR-200 family contributes to H19-mediated metastasis suppression in hepatocellular carcinoma. Carcinogenesis. 2013;34(3):577-86.

48. Zhu X, et al. Long noncoding RNA glypican 3 (GPC3) antisense transcript 1 promotes hepatocellular carcinoma progression via epigenetically activating GPC3. FEBS J. 2016;283(20):3739-54.

49. Wang $Y$, et al. The long noncoding RNA IncTCF7 promotes self-renewal of human liver cancer stem cells through activation of Wnt signaling. Cell Stem Cell. 2015;16(4):413-25.

50. Helming KC, Wang X, Roberts CWM. Vulnerabilities of mutant SWI/SNF complexes in cancer. Cancer Cell. 2014;26(3):309-17.

51. Chen ZZ, et al. LncSox4 promotes the self-renewal of liver tumourinitiating cells through Stat3-mediated Sox4 expression. Nat Commun. 2016;7:12598.

52. Yu Z, et al. SIX3, a tumor suppressor, inhibits astrocytoma tumorigenesis by transcriptional repression of AURKA/B. J Hematol Oncol. 2017;10(1):115.

53. Chen B. A novel long noncoding RNA IncWDR26 suppresses the growth and metastasis of hepatocellular carcinoma cells through interaction with SIX3. Am J Cancer Res. 2018;8(4):688-98.

54. Liu N, Liu Q, Yang X, et al. Hepatitis B Virus-Upregulated LNC-HUR1 Promotes Cell Proliferation and Tumorigenesis by Blocking p53 Activity. Hepatology. 2018;68:2130-44.

55. Liang WC, et al. LncRNA-NEF antagonized epithelial to mesenchymal transition and cancer metastasis via cis-regulating FOXA2 and inactivating Wnt/beta-catenin signaling. Oncogene. 2018;37(11):1445-56.

56. Wang $X$, et al. Long non-coding RNA DILC regulates liver cancer stem cells via IL-6/STAT3 axis. J Hepatol. 2016;64(6):1283-94.

57. Ding $L$, et al. Long noncoding RNA IncCAMTA1 promotes proliferation and Cancer stem cell-like properties of liver Cancer by inhibiting CAMTA1. Int J Mol Sci. 2016;17(10).

58. Sun QM, Hu B, Fu PY, et al. Long non-coding RNA 00607 as a tumor suppressor by modulating NF-kappaB p65/p53 signaling axis in hepatocellular carcinoma. Carcinogenesis. 2018;39:1438-46. 
59. Corbett AH. Post-transcriptional regulation of gene expression and human disease. Curr Opin Cell Biol. 2018;52:96-104.

60. Eulalio A, Huntzinger E, Izaurralde E. Getting to the root of miRNA-mediated gene silencing. Cell. 2008;132(1):9-14.

61. Hausser J, Zavolan M. Identification and consequences of miRNA-target interactions--beyond repression of gene expression. Nat Rev Genet. 2014; 15(9):599-612

62. Yuan JH, et al. The MBNL3 splicing factor promotes hepatocellular carcinoma by increasing PXN expression through the alternative splicing of IncRNA-PXN-AS1. Nat Cell Biol. 2017;19(7):820-32.

63. Yuan SX, et al. Long noncoding RNA DANCR increases stemness features of hepatocellular carcinoma by derepression of CTNNB1. Hepatology. 2016; 63(2):499-511.

64. Guo W, et al. ICAM-1-related noncoding RNA in Cancer stem cells maintains ICAM-1 expression in hepatocellular carcinoma. Clin Cancer Res. 2016;22(8): 2041-50.

65. Cui $\mathrm{M}$, et al. A long noncoding RNA perturbs the circadian rhythm of hepatoma cells to facilitate hepatocarcinogenesis. Neoplasia. 2015;17(1):79-88.

66. Li Y, et al. Long noncoding RNA IncARSR promotes doxorubicin resistance in hepatocellular carcinoma via modulating PTEN-PI3K/Akt pathway. J Cell Biochem. 2017;118(12):4498-507.

67. Yuan $\mathrm{JH}$, et al. A long noncoding RNA activated by TGF-beta promotes the invasion-metastasis cascade in hepatocellular carcinoma. Cancer Cell. 2014; 25(5):666-81.

68. Gauchotte $\mathrm{G}$, et al. Cytoplasmic overexpression of RNA-binding protein HuR is a marker of poor prognosis in meningioma, and HuR knockdown decreases meningioma cell growth and resistance to hypoxia. J Pathol. 2017;242(4):421-34.

69. Chou SD, et al. HSF1 regulation of beta-catenin in mammary cancer cells through control of HuR/elavL1 expression. Oncogene. 2015;34(17):2178-88.

70. Cao C, et al. The long intergenic noncoding RNA UFC1, a target of MicroRNA 34a, interacts with the mRNA stabilizing protein HuR to increase levels of beta-catenin in HCC cells. Gastroenterology. 2015;148(2):415-26 e18.

71. Zhang DY, et al. Identification and functional characterization of long noncoding RNA MIR22HG as a tumor suppressor for hepatocellular carcinoma. Theranostics. 2018:8(14):3751-65.

72. Reinstein $\mathrm{E}$, Ciechanover A. Narrative review: protein degradation and human diseases: the ubiquitin connection. Ann Intern Med. 2006;145(9): 676-84

73. Zhang $\mathrm{H}$, et al. The E3 ubiquitin ligase CHIP mediates ubiquitination and proteasomal degradation of PRMT5. Biochim Biophys Acta. 2016;1863(2): 335-46.

74. Ettenberg $\mathrm{S}$, et al. Cbl-b inhibits epidermal growth factor receptor signaling. Oncogene. 1999;18(10):1855-66.

75. Chen $\mathrm{H}$, et al. Long noncoding RNA LNC473 inhibits the ubiquitination of survivin via association with USP9X and enhances cell proliferation and invasion in hepatocellular carcinoma cells. Biochem Biophys Res Commun. 2018;499(3):702-10.

76. Zhu $P$, et al. Lnc-beta-Catm elicits EZH2-dependent beta-catenin stabilization and sustains liver CSC self-renewal. Nat Struct Mol Biol. 2016; 23(7):631-9.

77. Wang F, et al. Oncofetal long noncoding RNA PVT1 promotes proliferation and stem cell-like property of hepatocellular carcinoma cells by stabilizing NOP2. Hepatology. 2014;60(4):1278-90.

78. Xiao J, et al. LnCRNA HANR promotes tumorigenesis and increase of Chemoresistance in hepatocellular carcinoma. Cell Physiol Biochem. 2017; 43(5):1926-38.

79. Kikuchi A, Kishida S, Yamamoto $H$. Regulation of Wnt signaling by proteinprotein interaction and post-translational modifications. Exp Mol Med. 2006; 38(1):1-10.

80. Ding $\mathrm{C}-\mathrm{H}$, et al. The HNF1a-regulated IncRNA HNF1A-AS1 reverses the malignancy of hepatocellular carcinoma by enhancing the phosphatase activity of SHP-1. Mol Cancer. 2018;17:1.

81. Zhu P, et al. LncBRM initiates YAP1 signalling activation to drive self-renewal of liver cancer stem cells. Nat Commun. 2016;7:13608.

82. Ji J, et al. Long non-coding RNA Lnc-Tim3 exacerbates CD8 T cell exhaustion via binding to Tim-3 and inducing nuclear translocation of Bat3 in HCC. Cell Death Dis. 2018;9(5):478.

83. Fu X, et al. Linc00210 drives Wnt/beta-catenin signaling activation and liver tumor progression through CTNNBIP1-dependent manner. Mol Cancer. 2018;17(1):73.
84. Yuan SX, et al. Long noncoding RNA associated with microvascular invasion in hepatocellular carcinoma promotes angiogenesis and serves as a predictor for hepatocellular carcinoma patients' poor recurrence-free survival after hepatectomy. Hepatology. 2012;56(6):2231-41.

85. Huang JF, et al. Hepatitis B virus $X$ protein ( $H B X)$-related long noncoding RNA (IncRNA) down-regulated expression by HBx (Dreh) inhibits hepatocellular carcinoma metastasis by targeting the intermediate filament protein vimentin. Hepatology. 2013;57(5):1882-92.

86. Hacisuleyman E, et al. Topological organization of multichromosomal regions by the long intergenic noncoding RNA firre. Nat Struct Mol Biol. 2014;21(2):198-206

87. Xiang JF, et al. Human colorectal cancer-specific CCAT1-L IncRNA regulates long-range chromatin interactions at the MYC locus. Cell Res. 2014;24(5): 513-31

88. Mariner PD, et al. Human Alu RNA is a modular transacting repressor of mRNA transcription during heat shock. Mol Cell. 2008;29(4):499-509.

89. Guo H, et al. Modulation of long noncoding RNAs by risk SNPs underlying genetic predispositions to prostate cancer. Nat Genet. 2016;48(10):1142-50.

90. Jendrzejewski J, et al. The polymorphism rs944289 predisposes to papillary thyroid carcinoma through a large intergenic noncoding RNA gene of tumor suppressor type. Proc Natl Acad Sci U S A. 2012;109(22):8646-51.

91. Kim T, et al. Long-range interaction and correlation between MYC enhancer and oncogenic long noncoding RNA CARLo-5. Proc Natl Acad Sci U S A. 2014;111(11):4173-8.

92. Zheng J, et al. Pancreatic cancer risk variant in LINC00673 creates a miR1231 binding site and interferes with PTPN11 degradation. Nat Genet. 2016; 48(7):747-57.

93. Gao P, et al. Biology and clinical implications of the 19q13 aggressive prostate Cancer susceptibility locus. Cell. 2018;174(3):576-89 e18.

94. Hua JT, Ahmed M, Guo H, et al. Risk SNP-Mediated Promoter-Enhancer Switching Drives Prostate Cancer through IncRNA PCAT19. Cell. 2018;174: 564-75 e18.

95. Zhu Z, et al. An insertion/deletion polymorphism within RERT-IncRNA modulates hepatocellular carcinoma risk. Cancer Res. 2012;72(23):6163-72.

96. Tao R, et al. Association between indel polymorphism in the promoter region of IncRNA GAS5 and the risk of hepatocellular carcinoma. Carcinogenesis. 2015;36(10):1136-43.

Ready to submit your research? Choose BMC and benefit from:

- fast, convenient online submission

- thorough peer review by experienced researchers in your field

- rapid publication on acceptance

- support for research data, including large and complex data types

- gold Open Access which fosters wider collaboration and increased citations

- maximum visibility for your research: over $100 \mathrm{M}$ website views per year

At $\mathrm{BMC}$, research is always in progress.

Learn more biomedcentral.com/submissions 